\title{
Distribution of essential interactions in model gene regulatory networks under mutation-selection balance
}

\author{
Z. Burda ${ }^{1}$, A. Krzywicki ${ }^{2}$, O.C. Martin ${ }^{3,4}$ and M. Zagorski ${ }^{1}$ \\ ${ }^{1}$ Marian Smoluchowski Institute of Physics and Mark Kac Complex Systems Research Centre, \\ Jagellonian University, Reymonta 4, 30-059 Krakow, Poland \\ 2 Univ Paris-Sud, LPT ; CNRS, UMR8627, Orsay, F-91405, France. \\ 3 Univ Paris-Sud, LPTMS ; CNRS, UMR 8626, F-91405, Orsay, France \\ 4 INRA, CNRS, UMR 0320 / UMR 8120 Génétique Végétale, F-91190 Gif-sur-Yvette, France
}

(Dated: March 1, 2022)

\begin{abstract}
Gene regulatory networks typically have low in-degrees, whereby any given gene is regulated by few of the genes in the network. They also tend to have broad distributions for the out-degree. What mechanisms might be responsible for these degree distributions? Starting with an accepted framework of the binding of transcription factors to DNA, we consider a simple model of gene regulatory dynamics. There, we show that selection for a target expression pattern leads to the emergence of minimum connectivities compatible with the selective constraint. As a consequence, these gene networks have low in-degree, and "functionality" is parsimonious, i.e., is concentrated on a sparse number of interactions as measured for instance by their essentiality. Furthermore, we find that mutations of the transcription factors drive the networks to have broad out-degrees. Finally, these classes of models are evolvable, i.e., significantly different genotypes can emerge gradually under mutation-selection balance.
\end{abstract}

PACS numbers: 87.16.Yc, 87.18.Cf, 87.17.Aa

\section{INTRODUCTION}

It is by now well established that complex organisms typically do not have many more genes than less complex ones. Because of this, the paradigm for thinking about biological complexity has shifted from the number of genes to the way they work together: higher complexities might be associated with a greater proportion of regulatory genes. In particular, there are strong indications in eukaryotes and prokaryotes that for increasing genome size the number of regulatory genes grows faster than linearly in the total number of genes [1, 2]. Hence it is appropriate to consider biological complexity in the framework of interaction networks. This shift from components to the associated interactions has received increasing attention in many scientific communities, with applications ranging from network biology to sociology. The relevance of this conceptual framework for biology has been repeatedly emphasized and has benefited from inputs from other fields and from statistical physics in particular [3, 4]. We will therefore freely use the network terminology, refering to nodes, their degrees, distinguishing between in and out-degrees etc.

From studies that strive to unravel gene regulatory networks (GRN), several qualitative properties transpire: (i) a given gene is generally influenced by a "small" number of other genes (low in-degree of the network of interactions when compared to the largest possible degree [5, 6] ); (ii) some genes are very pleiotropic (the out-degree of some nodes of the network can be high, leading to degree distribution with fat tails or even possibly scale-free behavior [4, 6]); (iii) GRN seem to be robust to change (e.g., to environmental fluctuations or to mutations), a feature that is also found at many other levels of bio- logical organisation $[7-10]$. A simple way to build robustness into a network is to have rather dense connections, effectively incorporating redundancy, either locally or globally. Furthermore, the number of networks having $m$ interactions grows very quickly with $m$. Thus when modeling GRN, the network realizations that perform a given regulatory function are dominantly of very high degree. However this is not the case experimentally, at least with respect to the in-degree, and so models so far have had to build-in limitations to the accessible connectivities [11 13]. In this work we show that such shortcomings of models are overcome if one takes into account the known mechanisms underlying genetic interactions: gene regulation is mediated via the molecular recognition of DNA motifs by transcription factors, and this leads to biophysical constraints on interaction strengths. Within this relatively realistic framework, we shall see that networks under mutation-selection balance: (i) are driven to be parsimonious (the essential interactions are sparse) for the in-degree; (ii) can have broad distributions for the out-degree which is unconstrained; (iii) can evolve to very different realizations while preserving their function.

We begin by explaining the mechanisms incorporated into the model, in particular the determinants of the interactions. We follow standard practice [14 16] when modeling interactions between DNA binding sites and transcription factors (TF): the affinity depends on the mismatch between two character strings. We also specify how gene expression dynamics depend on these interactions and what "function" the networks must implement. The main difference between our approach and previous work is the introduction of a molecular definition of genotypes; this more realistic setting means that mutations are no longer ad-hoc and interestingly this difference leads to all the generic properties listed above. 
In fact, we keep our model as simple as possible to drive home the fact that all these properties emerge quite inevitably once such a setting is used. Clearly, our choice of a "minimal" model means that we must focus on qualitative aspects of the problem without attempting to reproduce specific experimental data.

After giving the general framework, we present some of the mathematical and computational tools we use to analyze the model. First, we demonstrate that selection (the constraint of having a given function or "phenotype") makes the networks' connectivity be close to minimal, i.e., networks are as sparse as they can be subject to maintaining their function. The "spontaneous" appearance of sparsity is due to the fact only a tiny (negligible) fraction of functional genotypes correspond to non-sparse networks. This entropic property is in strong contrast to what happens in models formulated in terms of networks (rather than in terms of microscopic genotypes). A consequence of this sparsity is that the genotypes are quite robust to mutations [9, 17]: only those few binding sites that are "effectively" used are fragile, mutations of the other (little used) binding sites have almost no effect. Thus robustness to mutational changes is very high for most binding sites while the "essential" interactions have much lower robustness; robustness is heterogeneously distributed in the network. Second, we find that the network out-degree is unconstrained, but that under evolutionary pressure coming from mutation-selection balance, broad out-degree distributions are favored. Implications of our work are developed in the discussion; in particular, a consequence is that redundant interactions are rapidly eliminated under evolution if no new function arises which might change the selection pressure.

\section{THE MODEL}

\section{A. Model genealogy}

Gene regulatory networks play an essential role in cellular dynamics. A question of major interest is how cells maintain their integrity; this includes stabilizing gene expression in the presence of environmental or genetic perturbations [18], actively maintaining oscillatory dynamics as in circadian clocks [19], or responding transiently and in a timely way to external signals [20]. In the majority of studies tackling these issues theoretically, one attempts to adjust model or kinetic parameters in gene circuits to obtain a GRN with the desired properties. Our focus here is different: we ask how functionality constrains network structure. Answering this requires considering the "space" of all functional GRN and determining the generic properties arising in this space. In particular, we shall be interested in the topological features and the robustness of these GRN.

The use of a space of GRN to propose "design principles" of gene regulation has been exploited previously by several groups [9, 11]. Such a paradigm enables one to put forward certain generic behaviors, and it is often complementary to other approaches. Perhaps the most widespread such framework was made popular by Stuart Kauffman (see 11] and references therein) using random boolean networks. Each node of the network is associated with a gene and its expression level which, by assumption, is a boolean variable ( $1 / 0$ for on/off) representing the possible two extreme expression levels of the gene. The gene expression dynamics at each node is taken as a random boolean function of its inputs; these inputs are the gene expression levels of the nodes it is directly connected to. Gene expression patterns or "phenotypes" are then represented by $N$-dimensional vectors with boolean components. Among other subjects, this framework was used to study a number of generic properties arising in this ensemble such as robustness to noise and mutations, evolutionary paths in classes of fitness landscapes and consequences for network topology.

Kauffman's approach led to the creation of entire familes of boolean models. Further steps were made by people trying to get additional insight by putting more knowledge into the boolean functions. Many works did this by focusing on "threshold" networks which had already been widely used in neural network modeling. There, the random boolean functions for each node are replaced by an "integrate and fire" type relation. To this end, one introduces an $N \times N$ matrix of inter-gene interactions [21] where $W_{i j}$ is the strength of the interaction of the $j$-th gene on the $i$-th one. (Notice that this matrix can be regarded as representing a directed weighted graph.) Typically these models remain boolean, so that the "output" is "on" if and only if the incoming (integrated) signal is above a threshold; the framework can then be considered as a refinement of Kauffman's. Models of this class have also been very popular, and have been used to study robustness, evolvability and other "generic" properties [22 26]. However, their main shortcoming is that the $W_{i j}$ weights are introduced in an adhoc way, with the consequence that any evolution of these networks is also arbitrary. In particular, one can deplore that these weights are in no way connected to known features of the underlying microscopic processes giving rise to genetic interactions.

Although we conceptually borrow much from the work of our predecessors, we also depart considerably from these earlier studies: we abandon the boolean approximation, we adapt known thermodynamical considerations to formulate our transcriptional dynamics, and we make use of the present knowledge of molecular processes to introduce in a realistic fashion interaction weights $W_{i j}$ and their dependence on mutations.

\section{B. Genotypes}

As already mentioned, GRNs are often represented by a directed weighted graph, i.e., by an $N \times N$ matrix $\left\{W_{i j}\right\}$, where $N$ is the number of genes belonging to 
the GRN. The $\left\{W_{i j}\right\}$ matrix can a priori be quite arbitrary. In complete generality, the products of the $N$ genes can have regulatory influences on the same set of genes (retroaction) and possibly also have some "downstream" consequences on other genes that do not code for TF. However the consequences of these last effects can be ignored for our purposes since they lead to no feedback on the $N$ "core" genes. The focus of all this paper is thus on such a core GRN, containing only TF coding genes. Note that these restricted networks have similar statistical properties to the unrestricted ones, namely sparsity, very low values for the in-degree, and a fat tail for the out-degree distribution [6].

Since it is the affinity between the transcription factor $j$ and its binding site (a DNA sequence) in the regulatory region of gene $i$ that microscopically controls the level of transcription, we want $W_{i j}$ to be a measure of this affinity. An affinity depends on the binding free energy which itself determines the frequency with which these two molecules will be bound rather than unbound. Following standard practice, we represent each $\mathrm{TF}$ as well as each binding site by a character string of length $L$; we also impose these characters to belong to a 4 letter alphabet in direct analogy with the four bases of DNA. The list of these strings then defines the molecular genotype of the system under consideration.

How can one connect this molecular genotype to a set of interactions $W_{i j}$ in the GRN? Building on the well known work of Berg and von Hippel [14], we assume for the sake of simplicity that the free energy of one TF molecule bound to its target is, up to an additive constant, equal to $\varepsilon d_{i j}$, where $d_{i j}$ is the number of mismatches between the strings representing the $j$-th TF and its binding site in the regulatory region of gene $i$. The parameter $\varepsilon$ is the penalty for each mismatch, i.e., the contribution to binding free energy in units of $k_{B} T$ where $k_{B}$ is the Boltzmann constant and $T$ is the temperature in degrees Kelvin. Experimentally, $\varepsilon$ is inferred to have values between one and three if one thinks of each base pair of the DNA as being represented by one character [27-29]. Note also that comparing to the typical number of base pairs found in experimentally studied binding sites leads to $10 \leq L \leq 15$. Given this framework, we now define the "interaction strengths" $W_{i j}$ arising in such a GRN via the Boltzmann factor

$$
W_{i j}=e^{-\varepsilon d_{i j}} / Z
$$

where $Z$ is a normalization (actually a partition function). If there were just one transcription factor molecule of type $j, W_{i j}$ would be the probability to find that molecule bound onto its binding site in the regulatory region of gene $i$. Gerland et al. [16] have shown that the term $Z$ in Eq. (1) is in practice close to 1 and that the probability of finding a particular transcription factor molecule bound at a given binding site is low.

If we consider the space of all genotypes, that is all possible character strings in our model with equal probability, the a priori distribution (coming from random strings) of the mismatch $d$ for any given pair $(i, j)$ is binomial:

$$
p(d)=\left(\begin{array}{l}
L \\
d
\end{array}\right)(1 / 4)^{L-d}(3 / 4)^{d} .
$$

Then for the biologically realistic values of $L$ mentioned above, using for instance Stirling's formula, we have

$$
p(d) \sim \frac{3^{d}}{d !} \frac{L^{d}}{4^{L}} \ll 1 \text { when } d \ll L .
$$

Hence small mismatches are very improbable, a fact that will be of utmost importance later on.

\section{Occupation of the binding sites}

Suppose, that there are $n_{j}$ TF molecules of type $j$ that can bind to a site in gene $i$ 's regulatory region; given that this site can be occupied only by one TF molecule at a time, it is necessary to take into account the possible competition. Using the fact that $Z$ in Eq. (1) is close to 1, it is possible to approximate the occupation probability of the binding site by [16]:

$$
P_{i j}=\frac{1}{1+1 /\left(n_{j} W_{i j}\right)} .
$$

In the following, we denote by $n$ the maximum number of TF molecules that can arise when a gene is fully "on", taking for simplicity this maximum to be independent of $j$. Biologically, $n$ is known to have a wide range, going from of order unity to many thousands. The lower value comes from some measurements of the multiplicity of transcripts [30, 31], while the higher value comes from other measurements of the numbers of transcription factor molecules [32]. For our study, we consider several values of $n$, reporting on the range $10 \leq n \leq 10^{4}$. We also considered smaller values for $n$ and found little change, but when $n$ is of order 1 , our "mean field" framework which neglects fluctuations can no longer be justified.

Setting $n$ to be $j$-independent is a rather strong assumption. It is made here for the sake of simplicity, to avoid a proliferation of free parameters. The implication is that we abandon any attempt to introduce external control factors. For example, it is known that the probability of a CAP molecule to attach to DNA and recruit a polymerase depends not only on the number of these molecules but on the concentration of glucose 33. By the same token, including co-factors or repressors or modeling more refined feedback circuits is beyond the scope of this paper.

\section{Phenotypes}

Call $S_{j}(t)$ the normalized level of gene $j$ 's products at time $t$, corresponding to a total number of TF 
molecules [34] equal to $n S_{j}(t)$. As already mentioned, we abandon the boolean approximation whereby a gene's expression level is either at its minimum or its maximum. Instead, we allow all intermediate values making $S_{j}(t)$ a continuous variable. In practice, we take all the $S_{j}(t)$ to lie in the interval $[0,1]$, corresponding to a minimum number of TF molecules of a given type equal to 0 and a maximum number equal to $n$. A short remark is in order for readers nourished with boolean models to avoid confusion: of course, instantaneously, a gene is either being transcribed (on) or not (off). However, these transcripts have to be translated into proteins (TFs) and these transcription factors will then have a certain half-life. Thus, it is necessary to distinguish the "effective" or average level of expression of a gene $j$ (here the number of $\mathrm{TF}$ molecules of type $j$ present) from the instantaneous transcription rate. In a model like this one, only the effective expression level is relevant.

At any given time, there is a vector of effective expression levels. In the next subsection we will define a deterministic dynamical system to model the time evolution of this vector. After possible transient behavior, at large times the set of expression levels $\left\{S_{j}\right\}_{j=1, \ldots N}$ may go to a (time-independent) steady state; we refer to this final vector as the "phenotype" of the GRN. It is also possible for the dynamics to go into a cycle (periodic behavior), but we shall not consider those GRNs in our analysis. Note that which case arises may depend on the initial expression levels.

GRNs enable the homeostasis of gene expression, allow for a response to a stimulus, or realize a new function such as cellular differentiation. In this work we consider that the "function" of our GRN is to bring the gene expression to a given pattern and maintain it there. This choice 21] is motivated from cases arising in early embryo development; there, the initial expression levels, $\left\{S_{j}^{(i n i t i a l)}\right\}_{j=1, \ldots N}$, are given (inherited during the formation of the egg). Then a network will "perform the desired function" if and only if, starting with the initial expression pattern, the vector of expression levels converges to a steady state which is close enough to a "target" pattern, which again must be given a priori. We will denote these target levels by $\left\{S_{j}^{\text {(target) }}\right\}_{j=1, \ldots N}$. Hereafter we loosely refer to a network as "viable" if it statisfies well this functional property, i.e., if its phenotype is sufficiently close to the target one.

\section{E. Expression pattern dynamics}

The dynamics of the $S_{j}(t)$ takes place on biochemical time scales (typically minutes). To model these dynamics, we consider that the normalized level $S_{i}(t+1)$ of the $i$-th gene at time $t+1$ is strongly associated with its transcription rate at time $t$. Clearly, that transcription rate depends on the degree to which the gene's regulatory region is occupied by TFs. To find the probability that a TF of type $j$ is bound to gene $i$ 's regulatory region, we use Eq. (4) but where the term $n_{j}$ is replaced by $n S_{j}(t)$ :

$$
P_{i j}=1 /\left(1+h / W_{i j} S_{j}(t)\right) .
$$

Here we have introduced $h=1 / n$ which plays the role of an effective threshold for the action of $W_{i j}$. This helps in comparing our results with those obtained with threshold models (including those from ref. 35] ).

To keep the framework simple, we shall suppose that gene $i$ 's transcription is "on" whenever at least one TF is bound within its regulatory region and otherwise it is "off". This is reminiscent of an "OR" logical gate whereby the output is on if and only if at least one of the inputs is on. The (normalized) mean expression level of a gene is then identified with the probability that transcription is on. Taking the TF occupancies in the regulatory region to be statistically independent, we then have

$$
S_{i}(t+1)=1-\prod_{j}\left(1-P_{i j}\right)
$$

Eqs. (6) and (5) define our discrete time transcriptional dynamics.

Two qualitative remarks are in order. First, we have taken the occupation probabilities of the different TFs to be independent; our framework thus ignores collective binding effects and in particular interactions between TF. Second, we have restricted the transcriptional logic to be of the "OR" type. Our TF thus act only as enhancers, never as repressors, and they do not cooperate using more complicated logic [36]. Since repressors are widespread in GRN, we have looked into generalizations of our model: we allowed repressor interactions while at the same time forcing multiple target expression patterns for a GRN to be viable. The resulting GRN are still sparse and their out-degree distribution still has a fat tail. However, since this paper's goal is to show how sparsity and high outdegrees emerge within minimal hypotheses, we postpone presenting these generalizations to a later work.

One can also ask whether our model is structurally robust. Any real expression dynamics will include fluctuations in the number of TF molecules [31, 37, 38] whereas our $S_{i}(t)$ dynamics are mean-field-like and thus neglect fluctuations. In general such an approximation can be justified when the number of molecules involved is large, i.e., in our case when $n$ is large. However, it is rarely clear a priori what is "large enough". Thus we have investigated the effects of adding noise to Eq. (6), letting the number of molecules $n S_{i}(t)$ become a Poisson random variable whose mean is given by that equation. Interestingly, none of the properties of the resulting GRN are significantly modified, even when going down to $n=10$. This can be understood qualitatively by considering the robustness of Eq. (5). $P_{i j}$ in that equation will be affected by noise in $S_{j}(t)$, but much less than by the modification of $W_{i j}$ induced by changing the mismatch $d_{i j}$ by one unit. One can then expect that high level of noise in the expression dynamics is unable to compensate the relevant mismatches present in the genotype. 
regulatory region of $\mathrm{N}$ sites

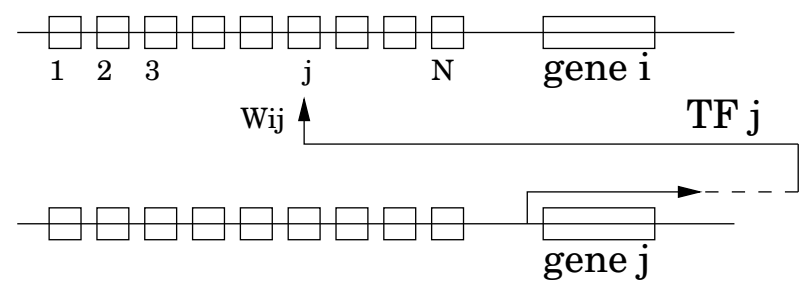

FIG. 1: Schematic representation of the regulatory region of gene $i$ : there are $N$ binding sites, each labeled by an index $j(1 \leq j \leq N)$. Represented is the interaction $W_{i j}$ mediated by the binding of TF $j$ to the $j$ 'th site of that region. The binding affinities depend on the mismatch between the string of length $L$ representing the $\mathrm{TF}$ and that representing the DNA of the corresponding binding site.

\section{IMPLEMENTATION}

To minimize the complexity of the model, we consider that each gene's regulatory region consists of $N$ putative binding sites, one for each of the $N$ types of TFs as illustrated in Fig. 1. If gene's $j$ normalized expression level is $S_{j}$, it will produce a certain number $n S_{j}$ of TF molecules of type $j$.

The genotype of one of our networks consists of many strings of length $L$ containing characters from a four letter alphabet. Explicitly, for each of the $N$ genes, one string encodes the TF and $N$ strings encode the DNA sequences of the different binding sites in the regulatory region of that gene. There is thus a total of $N(N+1) L$ characters specifying each genotype. This in fact defines the (discrete) space of all genotypes. However we are not interested in all genotypes, we want those that have "good" phenotypes. This leads us to define quantitatively a fitness for each genotype as follows.

Let us first define a "distance" between two phenotypes $\mathbf{S}$ and $\mathbf{S}^{\prime}$ using the differences in expression levels for each gene:

$$
D\left(\mathbf{S}, \mathbf{S}^{\prime}\right)=\sum_{i}\left|S_{i}-S_{i}^{\prime}\right|
$$

Algorithmically, we consider that the dynamical process Eq. (6) has reached a fixed point if $D(\mathbf{S}(t+1), \mathbf{S}(t))<$ $10^{-8}$. Given a phenotype, we take its fitness to be

$$
F(\mathbf{S})=\exp \left(-f D\left(\mathbf{S}, \mathbf{S}^{(\text {target })}\right)\right)
$$

where $f$ is a control parameter. A simple argument indicates that $f$ should be quite large: the maximum expression of gene $i$ (i.e., $S_{i}=1$ ) corresponds to the production of $n+O(\sqrt{n})$ TF molecules if one allows for Poisson noise. (This noise is unavoidable: diffusion as well as other processes necessarily lead to statistical fluctuations.) The relative fluctuation in $S_{i}$ is therefore $O(1 / \sqrt{n})$. Such a fluctuation must be innocuous for the cell and must not generate significant loss in fitness. Hence, one should roughly have $f / \sqrt{n}$ of order 1 or less, and thus $f=O(\sqrt{n})$ or less. In our simulations we use $f=20$; the results depend only very weakly on this choice provided $f$ is in the range 10 to 100 .

Given this definition of fitness, we can sample the fitness landscape of our system by Monte Carlo importance sampling. The goal is to bypass genotypes of low fitness and to focus instead on those genotypes whose phenotype is close to the target one. A standard approach to do this is to use the fitness as the measure to be used in the sampling: each genotype will appear with a probability proportional to its fitness. We do this by the Metropolis algorithm, producing a (biased) random walk in the fitness landscape which visits successive genotypes. At each step of the algorithm, we go to a neighboring genotype (in practice this is done by changing one character in the strings defining the genotype). Then the phenotype and fitness of this modified genotype is determined; the modified genotype is accepted or not, and the process is repeated. The acceptance procedure uses the Metropolis rule: if the fitness has increased, the modified genotype is accepted; if the fitness has decreased, the modified genotype is accepted with a probability given by the ratio of the new and old fitnesses. After many such steps, the algorithm will sample the space with the specified measure. In the next section, we shall consider the case where the TF character strings are fixed; the sampling of genotypes then restricts the modifications to arise only in the regulatory regions. In that case, we use "sweeps", a sweep being a succession of $L N^{2}$ attempted modifications.

For simplicity, we shall choose the $\left\{S_{j}^{(\text {initial })}\right\}_{j=1, \ldots N}$ and $\left\{S_{j}^{\text {(target) }}\right\}_{j=1, \ldots N}$ to be "on" (1) or "off" (0). If $S_{i=1, \ldots, N}^{(\text {initial })}$ and $S_{i=1, \ldots, N}^{\text {(target) }}$ are drawn at random, the number of components set to 1 will be approximately equal to that set to 0 when $N$ is large. For the results presented here, these numbers are set to $N / 2$ exactly as this reduces finite size effects in $N$. One can of course also use other choices for these numbers. To check the robustness of our model's properties, we have examined how the network connectivity is affected when we take $\mathbf{S}^{\text {(target) }}$ to have $N / 4$ or $3 N / 4$ components set to 1 instead of the $N / 2$ used throughout this paper. Interestingly, in all cases, we find that the same qualitative properties emerge, namely that for each gene that is "on" in $\mathbf{S}^{\text {(target) }}$ there is one incoming essential interaction (as defined later), while the out-degree distribution of the network has a fat tail. Coming back to our present choice motivated by drawing the expression vectors at random, we see that because of the permutation symmetry of the model, one can always swap the indices so that $S_{i}^{(\text {initial })}=1$ for $i \leq N / 2$ and 0 otherwise; furthermore we also impose without loss of generality $S_{i}^{(\text {target })}=1$ for $N / 4<i \leq 3 N / 4$ and 0 otherwise. Notice that $\sum_{i} S_{i}^{(\text {initial })}=\sum_{i} S_{i}^{(\text {target })}=N / 2$ and $\sum_{i} S_{i}^{(\text {initial })} S_{i}^{(\text {target })}=N / 4$. 


\section{BINDING SITE MUTATIONS AND SPARSITY}

\section{A. The emergence of a viable genotype}

We start by considering the TF encodings as given and fixed. The justification for this comes from the fact that because $\mathrm{TF}$ are typically pleiotropic, they are subject to strong stabilizing selection; they are thus generally thought to evolve slowly, while DNA regulatory regions typically have a high level of polymorphism and evolve more quickly [39]. In this and the next subsection, we adopt, as a first approximation, the assumption that the genes (and thus the TF they code) are fixed whereas the strings of characters representing the DNA binding sites are unconstrained. A consideration of the effects of TF mutations is postponed to the following section.

As already mentioned, the genotype determines the list of weights $W_{i j}$, corresponding to a weighted oriented graph. The first problem we address is the emergence of a viable genotype starting from an entirely random genotype. This can also be done by taking the $W_{i j}$ 's using mismatches generated from the binomial distribution Eq. (2). Random genotypes will produce very poor (unfit) phenotypes, but if the system is allowed to evolve under a selection pressure on the fitness, then it is possible that the genotypes will undergo gradual improvement until they are viable (highly fit). In our system, we thus ask for a steady state pattern $\mathbf{S}$ and subject the genotypes to a selection, for instance a selection proportional to the fitness as given in Eq. (8). If such selection is done on small populations, the resulting dynamics is quite similar to what arises in the Metropolis random walk algorithm, so we have implemented this in silico evolution process using the Monte Carlo algorithm described in the previous section.

In effect, identifying each of the attempted changes in our algorithm as a mutation, we examined the change in the fitness with increasing number of mutations. The result of this simulation is shown in Fig. 2, where we plot the fitness (actually the distance $D\left(\mathbf{S}, \mathbf{S}^{(\text {target })}\right)$ ) versus the number of mutational sweeps for two typical realizations. It is instructive to follow such a trajectory in more detail. Let us rewrite $P_{i j}(t)(c f$. Eq. (15)) as

$$
P_{i j}(t)=S_{j}(t) /\left(S_{j}(t)+x_{i j}\right),
$$

where $x_{i j}=h / W_{i j}$. In a random system, the $W_{i j}$ 's are grouped around the average value

$$
\left\langle W_{i j}\right\rangle=\left(0.25+0.75 e^{-\varepsilon}\right)^{L} .
$$

When $L=12$ and $\varepsilon=2$, this quantity is fairly small, about $3.6 \times 10^{-6}$. Hence, for $h>10^{-4}$, all $x_{i j}$ have large values. Consequently, for the initial (random) genotypes, Eq. (6) yields $S_{i} \approx 0$ on the l.h.s., and this is of course a steady state expression pattern. Since, by construction, $\mathbf{S}^{(\text {target })}$ has $N / 2$ elements equal to 1 , all other being 0 , the distance is then $D \approx N / 2$. Such a situation may

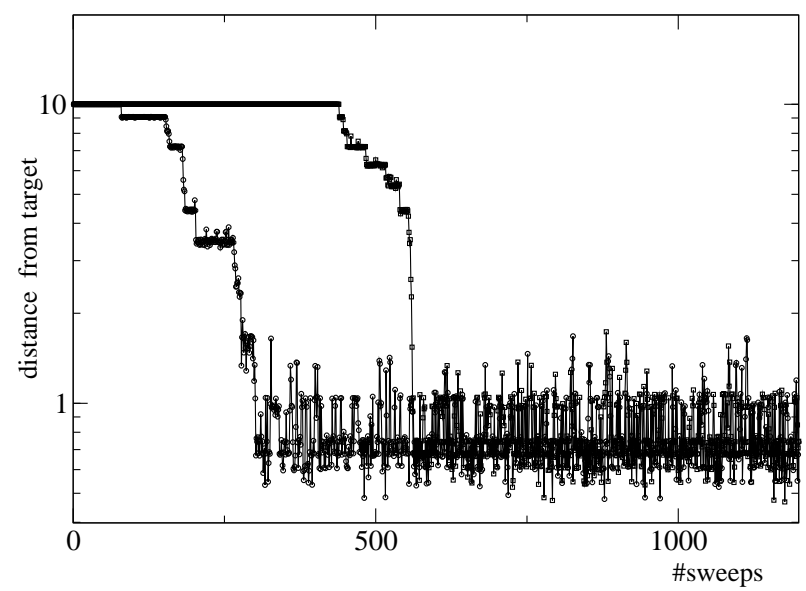

FIG. 2: Building up the stationary expression pattern $\mathbf{S}$ to $\mathbf{S}^{\text {(target) }}$, starting with a random genotype. Shown are two typical trajectories as a function of the number of mutational sweeps. Here $N=20, L=12, \varepsilon=2.0$ and $h=0.01$.

persist for a rather long time, until as the result of continuing mutations an unlikely event occurs: a diagonal element of the array $x_{i j}$, say $x_{k k}$, becomes small enough by chance. Then, one of the dynamical equations basically reduces to

$$
S_{k}(t+1) \approx S_{k}(t) /\left(S_{k}(t)+x_{k k}\right)
$$

with a non-trivial stationary solution $S_{k} \approx 1-x_{k k}$. For $x_{k k}=h \exp \left(\varepsilon d_{k k}\right)$ to be $O(1)$ or smaller, $d_{k k}$ must be less than $d_{h}=[\ln (1 / h) / \varepsilon]$, a value we call "critical" hereafter. For the parameter range under consideration, the generic result is that $S_{k}$ approaches values close to 1 and a relatively strong interaction appears. Such an interaction - which will turn out to be "essential" as explained soon - is characterized by a subcritical mismatch. In this situation, the distance $D$ to the target phenotype drops by an amount of order $O(1)$. Notice, that a subsequent significant increase of this distance is highly improbable because of the selection pressure: one unit of increase is counter-selected by a factor $\exp (-\sqrt{n})$ from what was explained in the previous section.

As the process continues with additional mutations, the increase in fitness dramatically accelerates. This is because the presence of every essential interaction has a double effect: it renders more probable the formation of new essential interactions which no longer need to be located on the diagonal, and it tends to lift up simultaneously several $S_{i}$ 's. The distance to the target phenotype then rapidly drops step by step until the system reaches a regime where $D$ fluctuates around a plateau value. For the choice of parameter values used in Fig. 2, this leads to $S_{i} \approx 0.94$ for each $i$ satisfying $S_{i}^{(\text {target })}=1$, while the remaining expression levels are negligibly small. From this "evolution" experiment, we see that our model allows for evolvable genotypes, in the sense that a new function can be acquired under realistic selection pressures if given enough time. 
It should be emphasized that a large auto-regulation $W_{k k}$ appears as the first noticeable evolutionary event (when a diagonal mismatch becomes small enough by chance). While such a direct auto-regulation is necessary to initiate the turning on of genes in our simulation of evolvability, it is not crucial later. We have thus measured the fraction of large auto-regulatory interactions at long times (when the Monte Carlo provides equilibrium samples of viable) to see if such interactions remain favored. The answer is no: the fraction of auto-regulatory interactions is no larger than expected by chance.

We should also mention that once a viable genotype has been found, in practice we find it to be viable also when using other choices for $\mathbf{S}^{\text {(initial) }}$ other than $\mathbf{S}=0$. (Thus the convergence of Eqs. (6) and (5) to the fixed point is largely independent of the starting expression pattern.) It seems that without introducing repressor interactions (which is beyond the scope of this paper), it is not possible to condition viability on $\mathbf{S}^{\text {(initial) }}$ in our model.

\section{B. Sparse essential interactions}

Remaining in the framework of having the TF encodings fixed, the Monte Carlo evolutionary dynamics just described will generate at large times an equilibrium distribution of fit (viable) genotypes. Recall that, because of the choice of our acceptance criterion, the algorithm will sample genotypes with a frequency proportional to their fitness. Hereafter we shall call this the space of viable genotypes. Viable genotypes define in essence a null hypothesis since that space determines the distribution of any GRN property arising from the sole constraint of function, i.e., the only constraint imposed on the GRN is that they be fit. We will now see that the vast majority of these GRN are in fact sparse, a feature that does not at all arise in GRN models that ignore the microscopic origin of the $W_{i j}$.

For our computational study, as suggested in Fig. 2. it is best to perform measurements every few hundred sweeps so that the data collected are not overly correlated. We typically used 10000 measurements separated by 100 sweeps. In Fig. 3 we show the distribution of the mismatch $d$ when there are $N=20$ genes, for increasing values of the "threshold" parameter $h$. At the low values of $h, d$ has a binomial distribution with a peak near $d=3 L / 4$ as expected. However at small $d$ one observes significant deviations. In fact, as $h$ increases, the viability constraint becomes more marked and the distribution becomes bimodal: a second peak appears at low mismatch values. Note that this peak shifts as $h$ increases, indicating that there are nearly perfect matches that appear in that regime.

Two remarks are in order: for intermediate values of $h$ it may happen that the peak at small $d$ extends over two neighbor values of $d$; the strong interactions then do not necessarily have the same strength. (Note that

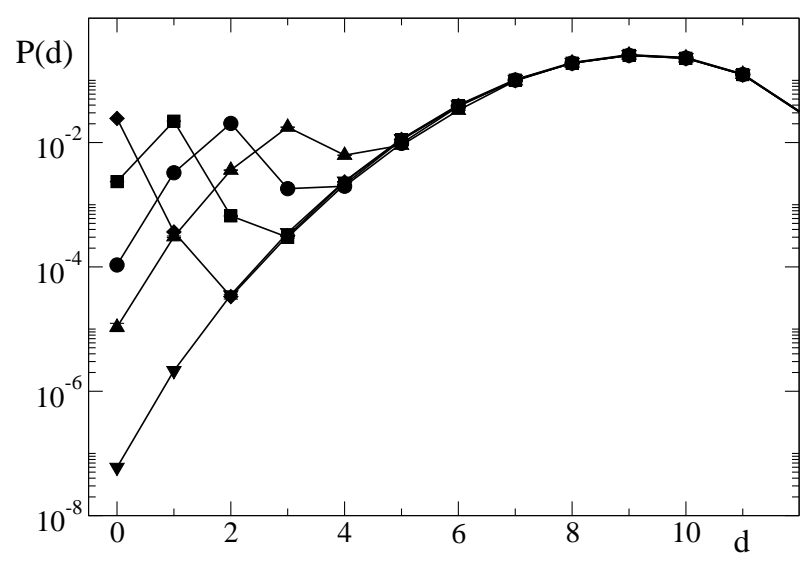

FIG. 3: Distribution of the Hamming distance between a TF and the receiving DNA site for $N=20, L=12, \varepsilon=2.0$ and for various values of the threshold parameter: (diamond) $h=0.1$, (square) $h=0.01$, (circle) $h=0.001$, (triangle up) $h=0.0001$, (triangle down) binomial. The lines are to guide the eye.

\begin{tabular}{|c|c|c|c|c|c|c|c|}
\hline 7 & 9 & 10 & 6 & 11 & 9 & 10 & 8 \\
\hline 9 & 7 & 9 & 10 & 10 & 9 & 9 & 9 \\
\hline 10 & 8 & 7 & 6 & 1 & 8 & 8 & 7 \\
\hline 8 & 9 & 10 & 8 & 10 & 1 & 8 & 10 \\
\hline 9 & 8 & 10 & 1 & 9 & 11 & 8 & 9 \\
\hline 11 & 12 & 9 & 7 & 11 & 1 & 8 & 10 \\
\hline 8 & 6 & 11 & 10 & 9 & 8 & 9 & 8 \\
\hline 10 & 10 & 9 & 10 & 8 & 11 & 9 & 9 \\
\hline
\end{tabular}

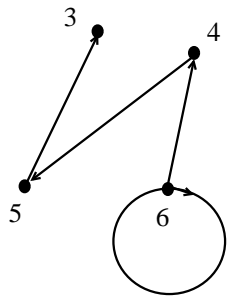

FIG. 4: Table of mismatches $d_{i j}$ between the $j$-th TF and its binding site in the regulatory region of gene $i$ for a toy GRN with $N=8$ (other parameters are as in Fig. 2). The mismatches corresponding to essential interactions are shown in boldface. In virtue of Eq. (11), the strength of the essential interactions is $\approx 0.135$ while the other interaction strengths are negligible (at most of order $10^{-6}$ ). On the r.h.s. we have drawn the network, keeping essential interactions only. The level of expression of the genes that are active in the target state is $\approx 0.926$ and that of the inactive ones ranges between $3 \times 10^{-6}$ and $6 \times 10^{-4}$.

because the $d$ take on discrete values, so do the $W_{i j}$.) Also, one could argue that the fitness parameter $f$ should be scaled like $\sqrt{h}$ when $h$ is changed. We have carried out calculations with such a rescaling but this change does not modify substantially the overall picture.

In Fig. 4 we show an illustrative case of the mismatches for a viable network with $N=8, \varepsilon=2.0$ and $h=0.01$. The bimodal nature of the mismatch distribution is clearly apparent; note in particular that the distribution at large $d$ is broad while that at small $d$ is 
narrow. Recalling that $W_{i j}=\exp \left(-\varepsilon d_{i j}\right)$, the interaction matrix can be considered to be sparse if we focus only on strong interactions. However doing so requires defining rather arbitrarily a distinction between strong and not strong $W_{i j}$. This difficulty is inherent to our framework where $W_{i j}$ is never 0 , in contrast to the situation in other gene network models where an interaction is present or absent.

Since reality does not follow a "present/absent" dichotomy for interactions, it is desirable to find a way to define mathematically a notion of sparsity in our context without too much arbitrariness. For this, let us consider not the weights themselves but their functionality. For a given genotype, call an interaction $W_{i j}$ "essential" if when setting it to zero viability is lost. With this definition, one can ask whether viable genotypes have sparse essential interactions, and in particular whether there are few essential interactions per row of the matrix $\left\{W_{i j}\right\}$. Notice that the number of active rows (i.e., having not too small expression levels) must be equal to the number of genes that are "on" in the target phenotype, $N / 2$ in our case, otherwise the fitness is far too low. We find that as soon as $h$ is not too small, there is almost always just one essential interaction per row as shown in Fig. 5 for $N=20$ and $L=12$. The same result holds for other relevant values of $N, n$ and $L$, suggesting that within our model, the drive towards sparse interactions arises in regimes of biological relevance.

We also considered a stronger measure of essentiality: we asked that viability be lost when the interaction's mismatch is increased by one. Remarkably, the rule "one essential incoming interaction per gene" generally held here too. Thus mutations in these interactions are typically deleterious, while mutations in the vast majority of the other interactions have no consequence on the fitness. This shows that mutational robustness is very heterogeneously distributed among the interactions in the network. The average robustness of fitness with respect to binding site mutations, $R_{b s}$, is readily estimated: there are $N / 2$ sensitive interactions out of $N^{2}$, hence one expects $R_{b s} \approx 1-1 / 2 N$. And indeed we find $R_{b s}=0.977(2)$ for $N=20$ (with some weak dependence on $h$ in the third decimal).

A consequence of the above is that in each active row of a viable network the mismatch distribution is (semiquantitatively) well represented by the ansatz $(H(x)=1$ for $x>0$ and 0 otherwise):

$$
\tilde{p}(d) \approx \frac{N-1}{N} p(d)+\frac{1}{N} \frac{p(d) H\left(d_{h}-d\right)}{\sum_{m<d_{h}} p(m)}
$$

with a very simple interpretation: the shape of the probability distribution of any mismatch is essentially that without the viability constraint, but with an additional peak at small values of the mismatch. There is thus one "leading" mismatch taking care of most of the viability constraint, while the other mismatches behave approximately as if they were unconstrained.

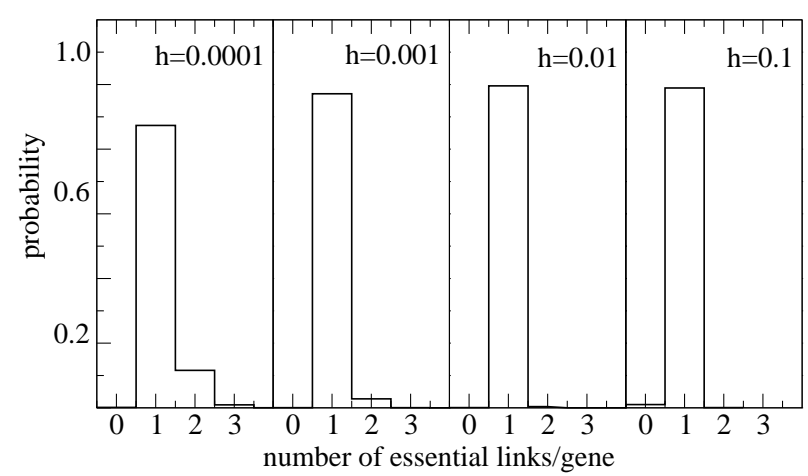

FIG. 5: Probability distribution of the number of essential interactions per row of the matrix specifying a viable network for $N=20, L=12, \varepsilon=2.0$ and a range of values of $h$.

It is interesting to see how the essential interactions are distributed among the columns of $\left\{W_{i j}\right\}$. First of all, they may appear in $N / 2$ columns only (in our example). This is an immediate consequence of the target phenotype being a fixed point of the dynamical process Eq. (6). A simulation yields the result that, as one might expect, the $N / 2$ essential interactions are distributed completely randomly among the $N / 2$ active columns. Since the number of essential interactions in a column is a measure of the effective out-degree of the corresponding node of the GRN (in the theory of weighted networks one would rather refer to the strength of the network node), one readily convinces oneself that the out-degree distribution is binomial with probability parameter equal to $2 / N$. More generally, let $K$ be the number of active genes in $\mathbf{S}^{\text {(target) }}$. Viable GRN will typically have $K$ essential reactions, all connecting these active genes. Furthermore, these essential interactions define a subnetwork where the in-degree is almost always 1 while the out-degree has a binomial distribution that is well approximated by a Poisson law of mean 1 .

A long succession of binding site mutations can move an essential interaction from one column to another. A few hundred sweeps are sufficient for that. Measuring the moments of the out-degree distribution one can calculate the autocorrelation time of this process. It turns out to be roughly of the order of $10^{3}$ sweeps for $h=0.01$ and about $10^{2}$ sweeps for $h=0.001$. This implies that very different genotypes can arise under mutation-selection balance if given enough time; our GRN model thus allows for gradual change of genotypes while maintaining phenotypes, a characteristic of evolvability.

\section{TF MUTATIONS AND A POPULATION DYNAMICS APPROXIMATION}

Up to now we assumed that the strings of characters associated with TFs were fixed. Of course, the genes coding for TFs can mutate. These mutations have very specific consequences for the GRN: when a TF is modified 
in our model, a whole column of the matrix $\left\{W_{i j}\right\}$ is affected at once. There will thus be a selective pressure on networks depending on how their essential interactions are spread across different columns, and this will change the out-degree distribution.

Qualitatively, one expects broad distributions to be favored; indeed, consider mutating a TF coding gene. If the $\mathrm{TF}$ is associated with at least one essential interaction, then such a mutation is typically highly deleterious. Oppositely, if the TF has no essential interactions, the mutations will be innocuous. Hence, in the presence of TF mutations, there is a strong correlation between the robustness $R_{t f}$ to mutations in the different TFs and column "occupancy" by essential interactions (i.e., the essential out-degree, to be denoted $k_{\text {out }}$ ). Let $K$ be the total number of essential interactions (typically equal to $N / 2$ in the results presented here), and let $N_{\text {occupied }}$ $\left(N_{\text {empty }}\right)$ be the number of occupied (empty) columns. Of course $N_{\text {occupied }}+N_{\text {empty }}=N$. Since mutating essential interactions is highly deleterious, the average robustness with respect to mutating a TF coding gene is accurately given by $R_{t f}=N_{\text {empty }} / N$ and it is easy to convince oneself that $\left\langle k_{\text {out }}\right\rangle=K / N_{\text {occupied }}$ where the average $\langle\cdot\rangle$ is to be taken only over occupied collumns. After elementary algebra one gets

$$
R_{t f}=1-\frac{K}{N\left\langle k_{\text {out }}\right\rangle} .
$$

In the examples discussed in this paper, $K=N / 2$, and therefore

$$
R_{t f}=1-\frac{1}{2\left\langle k_{\text {out }}\right\rangle},
$$

a result fully confirmed by an explicit numerical simulation. Thus, GRN with smaller $k_{\text {out }}$ are disfavoured as soon as we allow TFs to mutate.

To reveal the effects of selection, we must work with a population of GRN under mutation-selection balance and see the networks with broad out-degree distributions out-compete those with more narrow distributions. A large scale simulation of such a population would provide the distribution of $k_{\text {out }}$. Here we choose a simplified approach based on effective evolutionary dynamics as follows. First, one can safely ignore all the inactive genes in $\mathbf{S}^{\text {(target) }}$, and we are left with $K$ different TFs. Second, for each of these $K$ genes, there will almost always be exactly one essential (incoming) interaction. We thus map each microscopic genotype to an effective genotype, specifying only the essential interactions, as can be justified from the decoupling of Eq. (12). Third, we consider a population of such effective genotypes undergoing mutation and selection. At the microscopic level, we showed in the previous section that mutations of the binding sites will lead to slow changes in the positions of the essential interactions. These changes arise at the time scale $\tau$ which empirically we found to be several hundred sweeps, each sweep corresponding to $L N^{2} \mathrm{mu}-$ tations. That time scale $\tau$ is very large for the population dynamics under consideration so for simplicity think of $1 / \tau$ as being infinitesimal. Forth, at each generation we evolve the population of (effective) genotypes: the individuals are in competition because mutations in the TF coding genes affect them differently. The relevant characteristic of each genotype is its mutational robustness $R_{t f}$ to mutations in these genes. Thus $R_{t f}$ acts as a fitness which is under selection, with large $R_{t f}$ being favored; in view of Eq. (14), this should tend to enhance large out-degrees.

To see how this transpires within a population genetics framework, we consider an effective model where an essential interaction is represented by a "ball", a column of $\left\{W_{i j}\right\}$ by a "box" and an individual (e.g. a cell) in the population by $K$ boxes. The population of such "cells" is studied numerically simulating the well-known Moran process [40] as follows. At each step, we take a random individual of the population; let $N_{\text {occupied }}$ be its number of genes with out-degree greater or equal to 1 . A selection process is applied to this individual. With probability $R_{t f}=1-N_{\text {occupied }} / K$ it is duplicated and another randomly chosen individual is removed from the population. Otherwise (and thus with probability $N_{\text {occupied }} / K$ ), one tries another random individual, and so forth. With this Moran process, the population size stays constant as the fitness rises and reaches a limit. Higher fitness (robustness $R_{t f}$ ) corresponds via Eq. (14) to a broader out-degree distribution, large degrees being favored.

The simulation of this simplified population model is much faster than if we were to use the microscopic genotypes. It is also far more intuitive: each effective genotype can be thought of as a way to put $K$ balls into $K$ boxes. Each box is a TF and the balls in that box give the number of essential interactions associated with that TF. In Fig. 6] we display the resulting out-degree distribution for $K=10,20$ and 40 . The initial condition on the simulation is that the balls are distributed at random among the $K$ boxes. At this point a few comments are in order:

- For the choice $1 / \tau=0$, the result of the Moran process may depend on the initial condition. Since, in our case, the distribution of essential interactions among columns of $\left\{W_{i j}\right\}$ generated by binding site and TF mutations is different, one may worry that the results shown in Fig. 6] change if one mixes individuals with binomial and flat ball distributions. We have found that mixing them in equal proportion leads to an out-degree distribution that has almost the same shape as in Fig. 6. except that it is shifted to the left by about 0.5 , a minor modification.

- In reality, $\tau$ is not infinite, so at each generation, one should allow, at the rate $1 / \tau$, balls to go from one box to another. We checked explicitly that hops of balls done with frequency $1 / 10000$, still much larger than is realistic, do not change the result for the out-degree distribution in a perceptible way. 


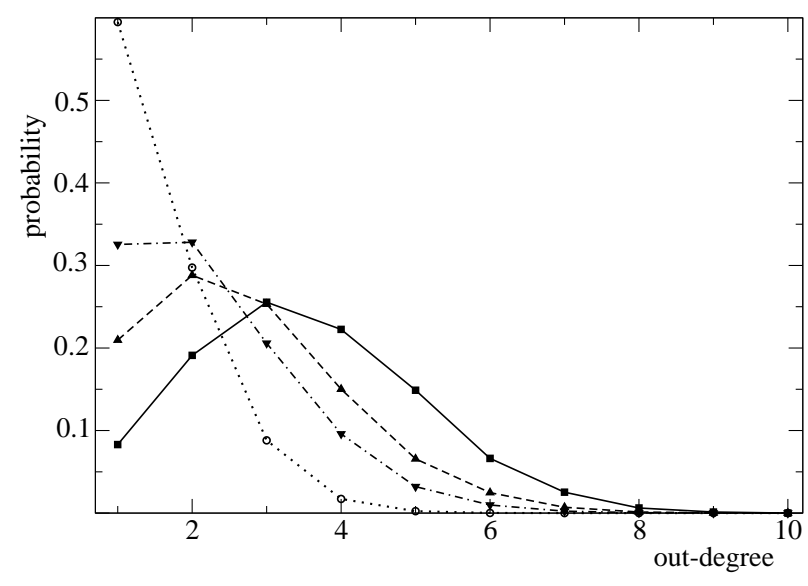

FIG. 6: Out-degree distribution within the simplified genotypes (see text) when using the Moran process. The population consists of 100000 genotypes and $K=10$ : squares, $K=20$ : triangles up, $K=40$ : triangles down (circles refer to the binomial distribution of mean 1 , which for these values of $K$ is almost $K$-independent).

\section{DISCUSSION AND CONCLUSION}

We considered a quite simple model of a gene regulatory network (GRN) in which function is identified with reaching given target gene expression levels. The key feature which sets our framework apart is the introduction of molecular genotypes which thereby specify interaction weights in the GRN. Within this setting, we have shown how a "viable" (i.e., having the desired function) GRN is progressively constructed under selection pressure, when one forces the phenotype to increase its "fitness" by approaching a previously defined optimal phenotype. We investigated the properties of networks in this model under the constraint that they be viable. We find that for a certain range of values of the model's parameters, the viability constraint leads to sparse GRN; we have quantified this through the sparsity of "essential" interactions. Interestingly, the effects of the viability constraint condense onto just a few of the interactions, the others being non-functional. As a result, nearly all mutations of the binding sites have no effect on the viability and so such sites have a very high mutational robustness. However, for those few sites which bear the essential interactions, the majority of mutations are deleterious so their mutational robustness is low. Thus in our GRN, the mutational robustness is extremely heterogeneous from site to site. In addition, any "redundant" interaction is expected to become lost under evolutionary dynamics since mutations will remove it and condense the burden of viability onto a smaller number of interactions. We have also studied the consequences of TF mutations for the fate of a population. In contrast to the behavior of the in-degree distribution which is "as narrow as possible", we find that the distribution of out-degrees develops a fat tail.

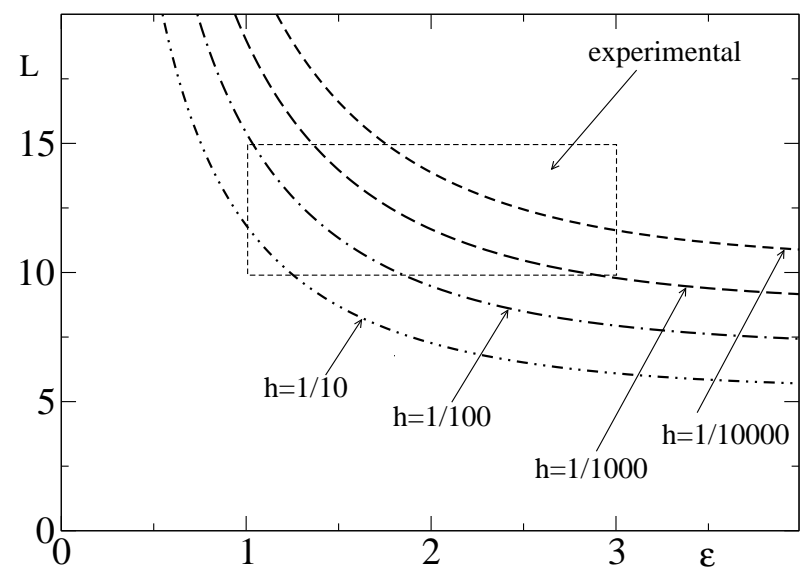

FIG. 7: The curves show the lower limit of the region where $h=10 N[0.25+0.75 \exp (-\varepsilon)]^{L}$ when $N=20$.

Although our modeling involves certain idealizations, its main characteristics are fairly realistic; in particular we have insisted on including interactions through the biophysical mechanism of molecular recognition and affinity. It is therefore quite striking that a reasonable GRN topology comes out very naturally in this framework. It should be clear that this success is a result of the combined effect of several causes: the viability constraint, the low probability of a small mismatch between $\mathrm{TF}$ and the binding site on the DNA, the size $L$ of this segment, the not-too-small spacing (in units of $k_{B} T$ ) between the energy levels that determine the strength of TF-DNA interactions, and finally the value of the threshold parameter $h$ itself which enters the dynamics of the gene expression levels ( $c f$. Eq. (6) ).

This overall picture corresponds to having all factors $P_{i j}$ in Eq. (6) be rather small except for the one which is associated with the essential incoming interaction. Such a scenario arises when the threshold $h$ is significantly larger than the sum of random $W_{i j}$ entering these factors (behaving, in practice, as if the viability constraint were absent). For a four letter code, assuming, as we do, that the binding energy is additive in mismatches and that every mismatch costs the same, one gets the condition

$$
h \gg N\left(0.25+0.75 e^{-\varepsilon}\right)^{L}
$$

Note that within a two letter code, the condition forces one to larger values of $L$ (close to 20) and thus beyond what is realistic biologically. Since the model parameters correspond to measurable quanties, it is appropriate to compare to biological values. According to Eq. (4) the probability that a TF occupies a DNA site is controlled by $h=1 / n$, where $n$ is the number of these molecules. We considered the range $1 / 10000<h<1 / 10$. Interpreting $\gg$ as "larger by one order of magnitude", i.e., by a factor 10, one gets an allowed region in parameter space as illustrated in Fig. [7.

This figure can be used to obtain the predicted domain of relevance: it is above the corresponding curves (taken 
at $N=20$ and illustrative values of $h$ ). We see that $\varepsilon$ and $L$ should not be too small. Moreover, it is gratifying that the experimental range of these parameters (indicated by a rectangle) is near the border and, most of it, within this region. The model would remain meaningful if $L$ and $\varepsilon$ were even larger. However, in the analogue of Fig. 3 , the point at $d=0$ would dominate strongly over the few neighboring $d$ points, and the GRN would be robust but not as evolvable [41, 42]. It is worth emphasizing that as the number $N$ of genes grows, it is necessary to increase slowly either $L, \varepsilon$ or $h$. $\varepsilon$ is constrained by biophysical processes and thus not evolvable, and $L$ seems the best candidate for the system to adapt to increasing $N$ [43]. Note nevertheless that the effects of growing $N$ are mild and that in practice regulation is modular, so effectively biological GRN have only modest values of $N$.

\section{Acknowledgments}

We thank V. Hakim, the late P. Slonimski and A. Wagner for helpful comments. This work was supported by the EEC's FP6 Marie Curie RTN under contract MRTNCT-2004-005616 (ENRAGE: European Network on Random Geometry), by the EEC's IST project GENNETEC - 034952 and by the Polish Ministry of Science Grant No. N N202 229137 (2009-2012). Project operated within the Foundation for Polish Science International Ph.D. Projects Programme co-financed by the European Regional Development Fund covering, under the agreement no. MPD/2009/6, the Jagiellonian University International Ph.D. Studies in Physics of Complex Systems. The LPT and LPTMS are Unité de Recherche de l'Université Paris-Sud associées au CNRS.
[1] E. V. Nimwegen, Trends Genet. 19, 479 (2003).

[2] M. Babu, N. Luscombe, L. Aravind, M. Gerstein, and S. Teichmann, Current Opinion in Structural Biology 14, 283 (2004).

[3] R. Albert and A. Barabasi, Rev. Mod. Phys. 74, 47 (2002).

[4] A. Barabasi and Z. Oltvai, Nat. Genet. 37, 937 (2005).

[5] D. Thieffry, A. Huerta, E. Perez-Rueda, and J. ColladoVides, Bio Essays 20, 433 (1998).

[6] G. Balazsi, A. Heath, L. Shi, and M. Gennaro, Mol. Syst. Biol. 4, 225 (2008).

[7] S. Bornholdt and K. Sneppen, Proc. R. Soc. Lond. B Biol. Sci. 267, 2281 (2000).

[8] J. Edlund and C. Adami, Artif. Life 10, 167 (2004).

[9] A. Wagner, Robustness and Evolvability in Living Systems (Princeton University Press, Princeton, NJ, 2005).

[10] M. Chaves, R. Albert, and E. Sontag, J. Theor. Biol. 235, 431 (2005).

[11] S. Kauffman, Origins of Order: Self-Organization and Selection in Evolution (Oxford University Press, Oxford, 1993).

[12] M. Aldana and P. Cluzel, Proc. Natl. Acad. Sci. 100, 8710 (2003).

[13] S. Kauffman and C. Peterson, Proc. Natl. Acad. Sci. 101, 17102 (2004).

[14] P. von Hippel and O. Berg, Proc. Natl. Acad. Sci. 83, 1608 (1986).

[15] O. Berg and P. von Hippel, J. Mol. Biol. 193, 723 (1987).

[16] U. Gerland, J. Moroz, and T. Hwa, Proc. Natl. Acad. Sci. 99, 12015 (2002).

[17] E. V. Nimwegen, J. Crutchfield, and M. Huynen, Proc. Natl. Acad. Sci. 96, 9716 (1999).

[18] C. Waddington, The Strategy of the Genes (Macmillan, New York, N.Y., 1957).

[19] A. Goldbeter, Biochemical Oscillations and Cellular
Rhythms: the Molecular Bases of Periodic and Chaotic Behaviour (Cambridge University Press, Cambridge, 1997).

[20] U. Alon, An Introduction to Systems Biology: Design Principles of Biological Circuits (Chapman and Hall, Boca Raton, FL, 2007).

[21] A. Wagner, Evolution 59, 1008 (1996).

[22] S. Bornholdt and T. Rohlf, Phys. Rev. Lett. 84, 6114 (2000).

[23] F. Li, T. Long, Y. Lu, Q. Ouyang, and C. Tang, Proc. Natl. Acad. Sci. 101, 4781 (2004).

[24] R. Azevedo, R. Lohaus, S. Srinivasan, K. Dang, and C. Burch, Nature 440, 87 (2006).

[25] S. Ciliberti, O. Martin, and A. Wagner, Proc. Natl. Acad. Sci. 104, 13591 (2007).

[26] R. Leclerc, Mol. Syst. Bio. 4, 213 (2008).

[27] A. Sarai and Y. Takeda, Proc. Natl. Acad. Sci. 86, 6513 (1989).

[28] G. Stormo and D. Fields, Trends in Biochem. Sci. 23, 109 (1998).

[29] M. Bulyk, P. Johnson, and G. Church, Nucl. Acids Res. 20, 1255 (2002).

[30] I. Golding, J. Paulsson, S. Zawilski, and E. Cox, Cell 123, 1025 (2005).

[31] A. Becskei, B. Kaufmann, and A. van Oudenaarden, Nat. Rev. Genet. 5, 101 (2004).

[32] J. Elf, G.-W. Li, and X. Xi, Science 316, 1191 (2007).

[33] S. Busby and N. Savery, In the online Encyclopedia of Life Sciences (2007).

[34] C. Pabo and R. Sauer, Ann. Rev. Biochem. 61, 1053 (1992).

[35] Z. Burda, A. Krzywicki, O. Martin, and Z. Zagorski, arXiv:0910.4077, unpublished.

[36] N. Buchler, U. Gerland, and T. Hwa, PNAS 100, 5136 (2003). 
[37] W. Blake, G. Balazsi, M. Kohanski, F. Isaacs, K. Murphy, Y. Kuang, C. Cantor, D. Walt, and J. Collins, Mol. Cell 24, 853 (2006).

[38] N. Barkai and B. Shilo, Mol. Cell 28, 755 (2007).

[39] M. Huynen and P. Bork, Proc. Natl. Acad. Sci. 95, 5849 (1998).

[40] P. Moran, The Statistical Processes of Evolutionary Theory (Clarendon Press, Oxford, 1962).
[41] J. Berg, S. Willmann, and M. Lassig, Evolutionary Biology 4, 42 (2004).

[42] V. Mustonen, J. Kinney, C. Callan, and M. Lassig, Proc. Natl. Acad. Sci. 105, 12376 (2008).

[43] A. Sengupta, M. Djordjevic, and B. Shraiman, Proc. Natl. Acad. Sci. 99, 2072 (2002). 